\title{
Multicriteria Design of Oversampled Uniform DFT Filter Banks
}

\author{
Ka Fai Cedric Yiu, Nedelko Grbić, Sven Nordholm, and Kok Lay Teo
}

\begin{abstract}
Subband adaptive filters have been proposed to avoid the drawbacks of slow convergence and high computational complexity associated with time domain adaptive filters. However, subband processing causes signal degradations due to aliasing effects and amplitude distortions. This problem is unavoidable due to further filtering operations in subbands. In this letter, the problems of aliasing effect and amplitude distortion are studied. Prototype filters which are optimized with respect to those properties are designed and their performances are compared. Moreover, the effect of the number of subbands, the oversampling factors and the length of the prototype filter are also studied. Using the multicriteria formulation, all Pareto optimums are sought via the nonlinear programming technique. We find that the prototype filter designed via the Kaiser window provides the best overall performance among the methods we studied. Also, there is a critical oversampling factor beyond which the improvement of performance is diminishing. Finally, if the length of the prototype filter increases with the number of subbands, an increase in the number of subbands will not deteriorate the performance.
\end{abstract}

Index Terms-Aliasing effect, amplitude distortion, filter bank, nonlinear programming, Pareto optimum, subband adaptive filter.

\section{INTRODUCTION}

A DAPTIVE filtering in subbands is an attractive alternative to full-band schemes in many applications. The DFT multirate filter banks are commonly used for efficient realization of the analysis and synthesis filter banks [1], [2]. However, the analysis of a signal into a subband representation and the synthesis back into its original full-band form has several difficulties. Noticeably, subband filterings introduce signal degradations which include signal distortions and aliasing effects [3]. It is well-known that a filter bank can be designed alias-free and the signals can be perfectly reconstructed when certain conditions are met by the analysis and synthesis filters. However, any filtering operation in the subbands may cause possible phase and amplitude changes and thereby altering the perfect reconstruction property. There are trade-offs in controlling both the

Manuscript received July 11, 2003; revised August 22, 2003. The work of the first and fourth authors was supported by the Research Grants Council of Hong Kong. The work of the second and third authors was supported by the Australian Research Council under Grant A00105530 The associate editor coordinating the review of this manuscript and approving it for publication was Prof. Victor A. N. Barroso.

K. F. C. Yiu is with the Department of Industrial and Manufacturing Systems Engineering, The University of Hong Kong, Hong Kong, China (e-mail: cedric@hkucc.hku.hk).

N. Grbić and S. Nordholm are with the Western Australian Telecommunications Research Institute (WATRI), The University of Western Australia and Curtin University of Technology, Perth, Australia.

K. L. Teo is with the Department of Applied Mathematics, The Hong Kong

Polytechnic University, Kowloon, Hong Kong, China.

Digital Object Identifier 10.1109/LSP.2004.826676 aliasing effect and the distortion level. Non-critical decimation has been suggested in [4] to improve the overall performance of the filter banks. Depending on the level of oversampling, the cost of computation also increases significantly.

In general, the filter bank design problem is a multicriteria decision problem, where the criteria are the level of distortion and the level of aliasing effect. A very sharp prototype filter will decrease the aliasing effect and distortion, but the length of the filter will be prohibitively long. In practice, the length of the prototype filter is often limited. Within this limit, the optimal filter must be sought. Methods have been proposed to minimize both criteria simultaneously, such as [5]. However, more flexible designs of the prototype filter have not yet been considered, and individual criterion has not yet been controlled directly. In this letter, these problems are addressed.

The performance of the filter bank depends on the choice of the prototype filter, the length of it, the number of subbands, and the oversampling factor. Here, we study the optimal designs for different combinations of parameters. To this end, the multicriteria formulation is used and is tackled via the nonlinear programming technique. This approach is versatile in the way that the specific performance of the filter bank can be imposed in advance. The aliasing and distortion levels can be controlled easily and the corresponding optimal weights can be sought. In this way, all the Pareto optimums can be calculated.

In assessing the performance, different prototype filter designs are studied here. These include the window method [6] with the Hamming window, the Kaiser window and the Dolph-Chebyshev window, and the minimax method. These methods are chosen since there are at most two free parameters which makes the filter design very simple. In addition, these window functions have different characteristics in controlling the main-lobe and side-lobes. The Hamming window is a standard window which adjusts the cutoff frequency only. The Kaiser window is a close approximation of the spheroidical wave functions and will concentrate the maximum energy to the centre. The Dolph-Chebyshev window tries to minimize the main-lobe width for a given side-lobe level. Among the windows investigated, we show that the Kaiser window gives the best overall performance with or without oversampling among the methods we study. Also, the effect of the oversampling factor, the number of subbands and the length of the prototype filter is also investigated.

\section{UNIFORM DFT MODULATED FILTER BANK}

In a typical analysis-synthesis DFT filter bank, two sets of $K$ filters form a uniform DFT analysis filter bank and synthesis filter bank. Assuming the same prototype filter with length $L$ is 
applied for both analysis and synthesis, the subband filters are related to the prototype FIR filter as

$$
H_{k}(z)=H\left(z W_{K}^{k}\right)=h^{T} \phi\left(z W_{K}^{k}\right) \quad k=0, \cdots, K-1
$$

where $W_{K}=e^{-j 2 \pi / K}, \boldsymbol{h}=[h(0), \cdots, h(L-1)]^{T}$ and $\phi(z)=$ $\left[1, z^{-1}, \cdots, z^{-(L-1)}\right]^{T}$. Each subband signal is decimated by a factor $D$. An implementation of this filter bank is depicted in Fig. 1. From Fig. 1, the input-output relationship can be derived and is given by

$$
\widehat{X}(z)=\frac{1}{D} \sum_{l=0}^{D-1} X\left(z W_{D}^{l}\right) \sum_{k=0}^{K-1} H\left(z W_{K}^{k} W_{D}^{l}\right) H^{*}\left(z W_{K}^{k}\right)
$$

where $W_{D}=e^{-j 2 \pi / D}$ and $W_{K}=e^{-j 2 \pi / K}$ and $*$ denotes conjugate. This may be rewritten as

$$
\widehat{X}(z)=\sum_{l=0}^{D-1} A_{l}(z) X\left(z W_{D}^{l}\right)
$$

where

$$
A_{l}(z)=\frac{1}{D} \sum_{k=0}^{K-1} H\left(z W_{K}^{k} W_{D}^{l}\right) H^{*}\left(z W_{K}^{k}\right) .
$$

If $A_{l}(z)=0$ for $l=1,2, \ldots, D-1$, and $A_{0}(z)=a z^{-b}$, for any $a, b$ where a $\neq 0$, we get a perfect reconstruction filterbank. However, any filtering operation in the subbands may cause possible phase and amplitude changes and thereby altering the perfect reconstruction property. Our main objective is to minimize both the aliasing power and the amplitude distortion by optimizing the prototype filter coefficients $\boldsymbol{h}$. The aliasing power and the amplitude distortion can be defined as

$$
A_{P}=\frac{1}{2 \pi} \int_{-\pi}^{\pi} \sum_{l=1}^{D-1}\left|A_{l}\left(e^{-j \omega}\right)\right|^{2} d \omega
$$

and

$$
A_{D}=\frac{1}{2 \pi D} \int_{-\pi}^{\pi}\left(1-\left|A_{0}\left(e^{-j \omega}\right)\right|\right)^{2} d \omega
$$

respectively. The aliasing effect is best understood by looking at Fig. 2 where an oversampling clearly reduces the aliasing effect due to the transition width of the prototype filter. When the oversampling factor increases, the lines of aliasing move apart to reduce the aliasing effect. In optimizing the prototype filter, simply minimizing a sum of both measures may result in performance skewing toward one extreme. There is no easy way to introduce any scaling factor to adjust such uneven performance. Because there are more than one objective in the design of the filter bank, it is basically a multicriteria design problem [7], [8]. When different restrictions are imposed to the criteria in the design process, a solution set can be derived in which all solutions are efficient, or Pareto optimum. In the present context, the set of weights $h^{*}$ is Pareto optimum if and only if there does not exist a set of weights $h$ such that

$$
A_{P}(\boldsymbol{h}) \leq A_{P}\left(\boldsymbol{h}^{*}\right) \quad \text { and } \quad A_{D}(\boldsymbol{h}) \leq A_{D}\left(\boldsymbol{h}^{*}\right)
$$

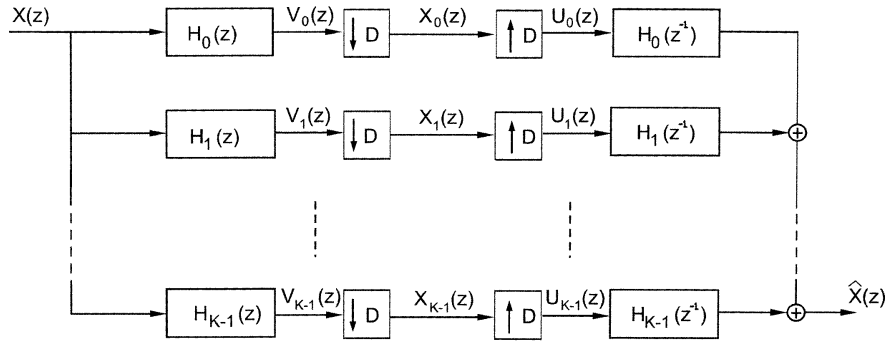

Fig. 1. Direct form realization of an analysis and synthesis filter-bank.

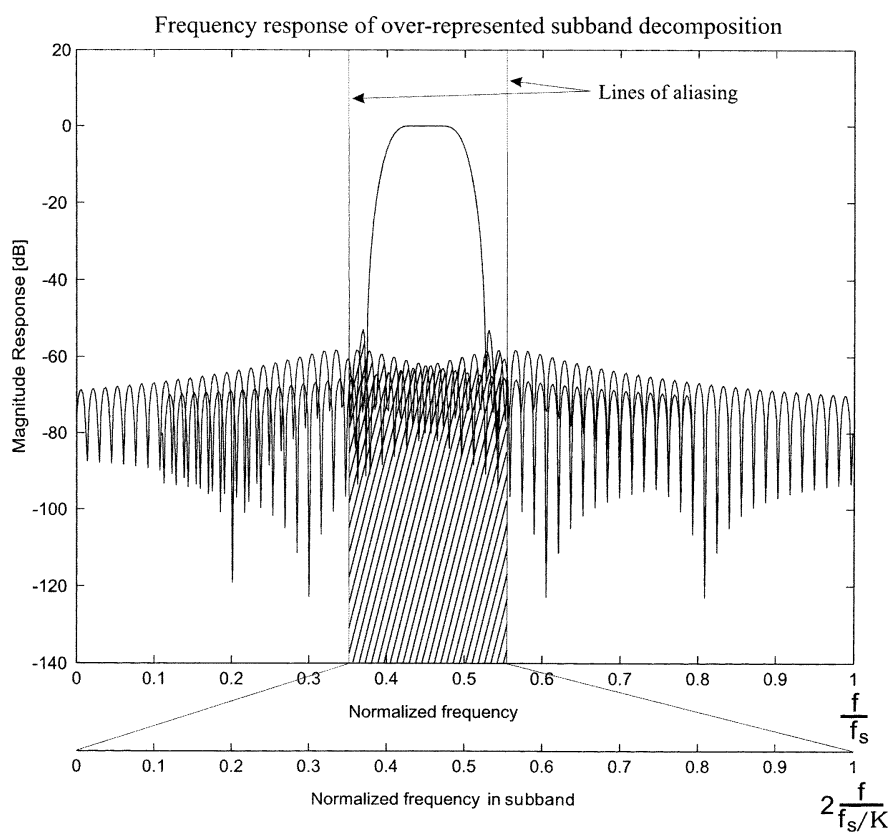

Fig. 2. Aliasing effect is reduced with oversampling (2x).

with strict inequality to at least one of the criteria. In order to solve for the Pareto optimum, some of the criteria can be formulated as constraints instead so that it becomes a nonlinear programming problem. An additional advantage of using this formulation is that the constraints can be adjusted freely to select the desired filter from the set of Pareto optimum solutions.

\section{Prototype Filter Design}

A typical nonrecursive causal prototype FIR filter can be defined by the transfer function

$$
H(z)=\sum_{n=0}^{L-1} h(n) z^{-n} .
$$

There are several ways to design this type of filters. One method is to use a window function. The filter coefficients $h(n)$ are given by the Window method as [9]

$$
h(n)=\frac{\sin \left(2 \pi f_{c}\left(n-\frac{L-1}{2}\right)\right)}{\pi\left(n-\frac{L-1}{2}\right)} w(n)
$$

where $w(n)$ is a window function. For a given number of subbands, $\mathrm{K}$, and a given decimation/interpolation factor, $\mathrm{D}$, and for a certain filter length, $\mathrm{L}$, we need to design the cutoff frequency $0<f_{c}<(1 / 2)$ and the corresponding window function. 


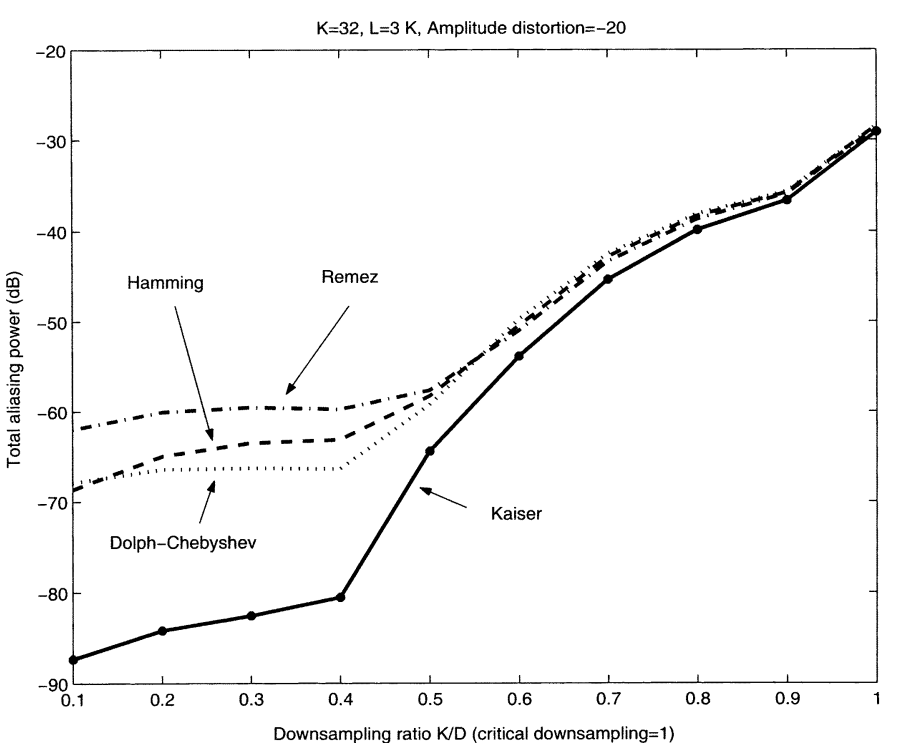

Fig. 3. Comparison for different design methods.

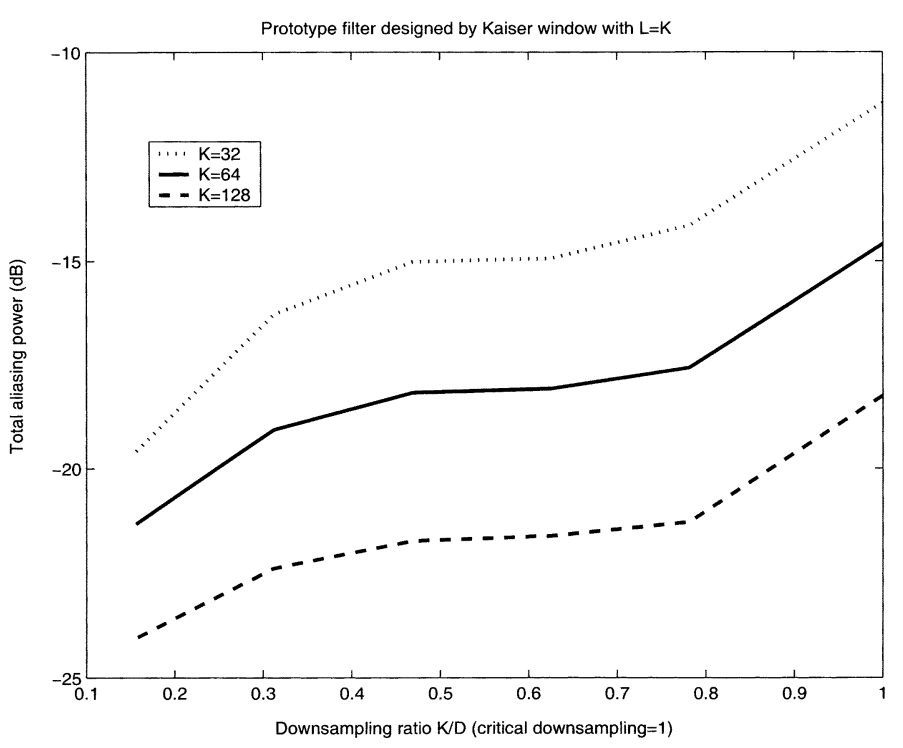

Fig. 4. Comparison for different number of subbands with $L=K$.

A simple popular window function is the Hamming window, and can be defined as [10]

$$
w(n)=\left\{\begin{array}{cl}
\alpha-(1-\alpha) \cos \frac{2 \pi n}{L-1}, & n=0,1, \cdots, L-1 \\
0, & \text { otherwise }
\end{array}\right.
$$

where $\alpha=0.54$. There is no additional parameter for this window function.

In case of the Kaiser window, there is an additional design parameter $\alpha$. Also, it can control the ripple ratio and the main-lobe width. This window is given by

$$
w(n)=\frac{\mathbf{I}_{o}\left(\alpha \sqrt{\left(\frac{L-1}{2}\right)^{2}-\left(n-\frac{L-1}{2}\right)^{2}}\right)}{\mathbf{I}_{o}\left(\alpha \sqrt{\left(\frac{L-1}{2}\right)^{2}}\right)}
$$

where $\mathbf{I}_{o}(x)$ is the zeroth-order modified Bessel function of the first kind.

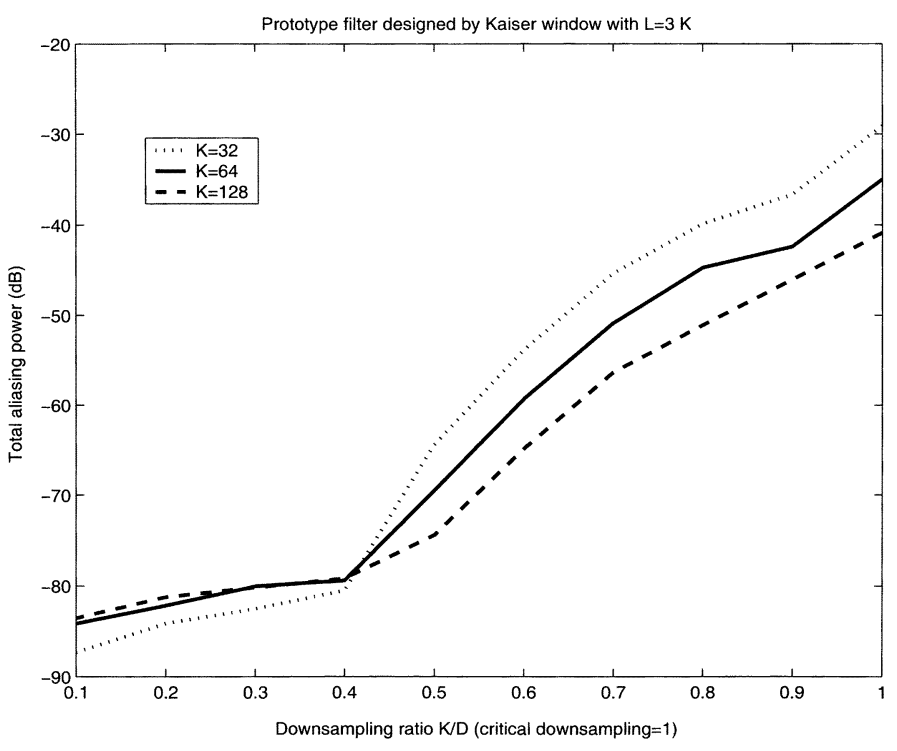

Fig. 5. Comparison for different number of subbands with $L=3 K$.

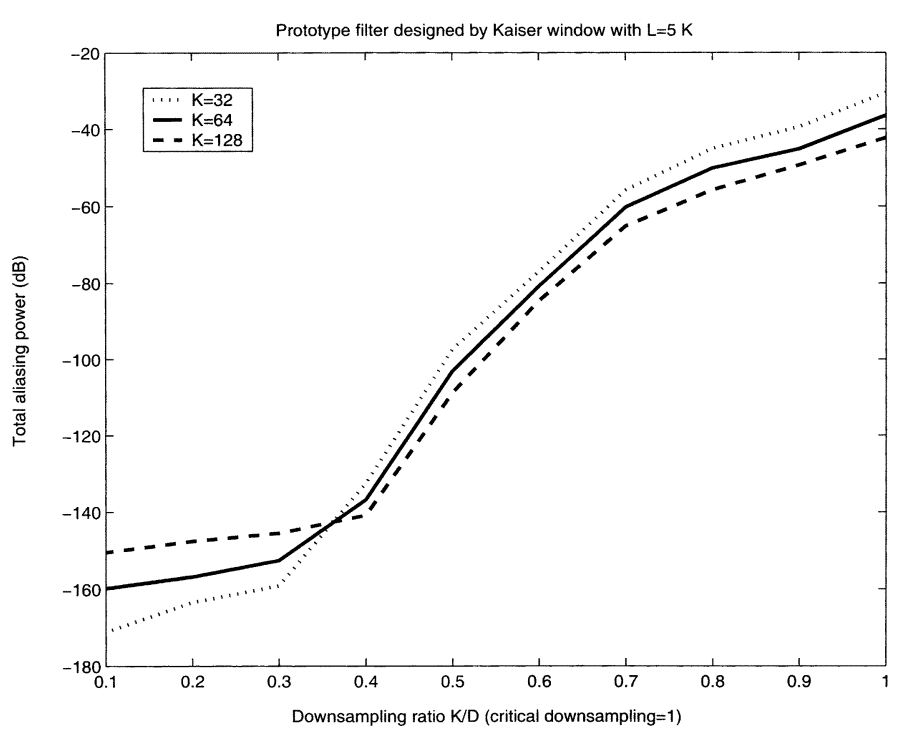

Fig. 6. Comparison for different number of subbands with $L=5 K$.

Another window which can vary the ripple ratio and main-lobe width is the Dolph-Chebyshev window, defined as

$$
w(n)=\frac{1}{L}\left[\frac{1}{r}+2 \sum_{i=1}^{\frac{(L-1)}{2}} T_{L-1}\left(\frac{x_{0} \cos i \pi}{L}\right) \cos \left(\frac{2 n \pi i}{L}\right)\right]
$$

where $r$ is the ripple ratio as a fraction, and

$$
x_{0}=\cosh \left(\frac{1}{L-1} \cosh ^{-1} \frac{1}{r}\right) .
$$

Function $T_{k}(x)$ is the $k$ th-order Chebyshev polynomial associated with the Chebyshev approximation for recursive filters and is given by

$$
T_{k}(x)= \begin{cases}\cos \left(k \cos ^{-1} x\right), & \text { for }|x| \leq 1 \\ \cosh \left(\cosh ^{-1} x\right), & \text { for }|x|>1\end{cases}
$$

The additional design parameter is therefore $r$ for this window. 
Apart from using the window method, it is also possible to use the minimax technique [9] instead of the least-squares technique to design (8). Once the cutoff frequency $f_{c}$ and the stop-band frequency $f_{s}$ is fixed, the minimax optimization problem can be solved quickly via the Remez exchange algorithm. The two design parameters for the minimax method are $f_{c}$ and $f_{s}$.

In order to control the aliasing effect and the amplitude distortion separately, the final optimal design problem can be formulated into an equivalent nonlinear programming problem as:

$$
\min _{\gamma} A_{P}(h(\gamma))
$$

subject to

$$
A_{D}(h(\gamma)) \leq \epsilon
$$

where $\epsilon$ is a pre-defined tolerance for the aliasing effect. The level of distortion can now be controlled freely. Note that $\gamma$ is one-dimensional for the Hamming window and two-dimensional for the other windows and the minimax method. One well-known method for solving this nonlinear programming problem is the sequential quadratic programming (SQP). This method is well described in [11] or [12] and will be applied here.

\section{EVALUATION RESULTS}

In the following, the amplitude distortion is restricted to be less than $-20 \mathrm{~dB}$ throughout. The first example is to assess different designs of the prototype filter and its implication to the aliasing effect for different oversampling factors. The result is depicted in Fig. 3. The first observation is that by increasing the oversampling factor, the optimal aliasing effect is decreasing. However, the decrease in the total aliasing power is diminishing when the oversampling factor increases. There is an critical oversampling factor, which resembles the point of inflection in the curve, beyond which there is very little improvement in the aliasing effect. The second important observation is that there is a big difference in performance between different windows, and the Kaiser window out-performed all the other methods for all oversampling factors. The oversampling Kaiser window provides a "don't-care" region in the pass-band of the prototype filter, which provides us with extra degrees of freedom in the design process. The Kaiser window is shown to make better use of these extra degrees of freedom. Moreover, the ripple ratio decreases with increasing $\alpha$. This parameter can be effectively employed to trade off the main-lobe width against the side-lobe amplitude. Finally, the minimax technique does not perform well in the filter bank design. This can be due to the fact that the cost function $A_{P}$ is defined in a least-squares sense, and is therefore not in favor of the minimax technique. Further study is required if the cost function is defined differently, possibly in a minimax sense.

In order to understand the influence of the prototype filter length and the number of subbands, we use the Kaiser window as a demonstration. As the number of subbands increases, the passband gets narrower. Thus, it is harder to maintain the low distortion level unless the length of the filter increases to allow for a narrower transition region. If the filter length is equal to the number of subbands, the result is shown in Fig. 4. In this case, it is simply a weighting of an FFT with different oversampling factors. It is interesting to see that as the number of subbands increases, there is almost a uniform improvement to the aliasing effect for all oversampling levels. If the filter length is fixed to be a constant multiple of the number of subbands, the results are depicted in Figs. 5 and 6. The optimal aliasing effects improve significantly. For sampling factors near to the critical one, the aliasing effect decreases uniformly with the increase of number of subbands. But this decrease diminished for higher oversampling factors. The critical oversampling factor can be read out from the graph which is roughly equal to the point with the highest curvature.

\section{CONCLUSIONS}

In this letter, the problem of filter bank design has been studied. The design problem has been formulated as a multicriteria optimization problem. Using the nonlinear programming technique, all the Pareto optimums can be solved and studied. Different window methods have been investigated. It turns out that among the methods we have studied, the Kaiser window gives the best performance with the lowest aliasing effect for a fixed amplitude distortion level. If the length of the prototype filter is proportional to the number of subbands, the optimal aliasing effect generally improves with the number of subbands for sampling factors close to the critical one. If oversampling is employed, we find that the improvement to the aliasing effect is diminishing. The critical oversampling factor is the point with highest curvature beyond which very little improvement in performance is observed.

\section{ACKNOWLEDGMENT}

The authors wish to thank the anonymous referees for their comments.

\section{REFERENCES}

[1] P. P. Vaidyanathan, Multirate Systems and Filter Banks. Englewood Cliffs, NJ: Prentice-Hall, 1993.

[2] S. Haykin, Adaptive Filter Theory. Englewood Cliffs, NJ: PrenticeHall, 2002.

[3] A. Gilloire and M. Vetterli, "Adaptive filtering in sub-bands with critical sampling: analysis, experiments, and application to acoustic echo cancellation," IEEE Trans. Signal Processing, vol. 40, pp. 1862-1875, Aug. 1992.

[4] W. Kellermann, "Analysis and design of multirate systems for cancellation of acoustic echoes," in Proc. ICASSP, 1988, pp. 2570-2573.

[5] J. M. de Haan, N. Grbić, I. Claesson, and S. Nordholm, "Design of oversampled uniform DFT filter banks with delay specification using quadratic optimization," in Proc. ICASSP, 2001.

[6] F. Harris, "On the use of windows for harmonic analysis with the discrete Fourier transform," Proc. IEEE, vol. 66, pp. 51-83, Jan. 1978.

[7] M. Zeleny, Multiple Criteria Decision Making. New York: McGrawHill, 1982.

[8] T. L. Vincent and W. J. Grantham, Optimality in Parametric Systems. New York: Wiley, 1981.

[9] T. W. Parks and C. S. Burrus, Digital Filter Design. New York: Wiley, 1987.

[10] J. G. Proakis and D. G. Manolakis, Digital Signal Processing: Principles, Algorithms, and Applications. Englewood Cliffs, NJ: PrenticeHall, 1996.

[11] R. Fletcher, Practical Methods of Optimization. New York: Wiley, 1987.

[12] K. L. Teo, C. J. Goh, and K. H. Wong, A Unified Computational Approach to Optimal Control Problems. New York: Longman, 1991. 